\title{
Vision Calibrates Sound Localization in Developing Barn Owls
}

\author{
Eric I. Knudsen and Phyllis F. Knudsen \\ Department of Neurobiology, Stanford University School of Medicine, Stanford, California 94305
}

This study demonstrates that continuous exposure of baby barn owls to a displaced visual field causes a shift in sound localization in the direction of the visual displacement. This implies an innate dominance of vision over audition in the development and maintenance of sound localization.

Twelve owls were raised from the first day of eye opening wearing binocular prisms that displaced the visual field to the right by $11^{\circ}, 23^{\circ}$, or $34^{\circ}$. The prisms were worn for periods of up to 7 months. Consistent with previous results (Knudsen and Knudsen, 1989a), owls reared with displacing prisms did not adjust head orientation to visual stimuli. While wearing prisms, owls consistently oriented the head to the right of visual targets, and, as soon as the prisms were removed, they oriented the head directly at visual targets, as do normal owls. In contrast, prism-reared owls did change head orientation to sound sources even though auditory cues were not altered significantly. Birds reared wearing $11^{\circ}$ or $23^{\circ}$ prisms oriented the head to the right of acoustic targets by an amount approximately equal to the optical displacement induced by the prisms. Birds raised wearing $34^{\circ}$ prisms adjusted sound localization by only about $50 \%$ of the optical displacement. Thus, visually guided adjustment of sound localization appears to be limited to about $20^{\circ}$ in azimuth.

The data indicate that when confronted with consistently discordant localization information from the auditory and visual systems, developing owls use vision to calibrate associations of auditory localization cues with locations in space in an attempt to bring into alignment the perceived locations of auditory and visual stimuli emanating from a common source. Vision exerts this instructive influence on sound localization whether or not visual information is accurate.

The auditory system localizes sound sources by associating sets of monaural and binaural cues with locations in space. For accurate localization, these cue-location associations must be calibrated. Experiments on humans and on barn owls indicate that the brain uses visual information to perform this calibration (Held, 1955; Kalil and Freedman, 1967; Knudsen et al., 1984a; Knudsen and Knudsen, 1985). When the correspondence of auditory cues with locations is altered, the accuracy of sound localization may be adjusted over time on the basis of spatial information provided by the visual system.

Received Feb. 6, 1989; revised Mar. 20, 1989; accepted Mar. 21, 1989.

We thank S. Esterly and B. Wallace for their comments on the manuscript. This work was supported by a neuroscience development award to E.I.K. from the McKnight Foundation and a grant from the National Institutes of Health (R01 NS 16099-08).

Correspondence should be addressed to Dr. Eric I. Knudsen, Department of Neurobiology, Stanford University School of Medicine, Sherman Fairchild Science Building, Room D200, Stanford, CA 94305-5401.

Copyright (C) 1989 Society for Neuroscience $0270-6474 / 89 / 093306-08 \$ 02.00 / 0$
A different kind of visual influence on sound localization is the psychophysical phenomenon referred to as "visual capture." Visual capture occurs when there is a discrepancy between the location of a visual object and the location of a logically consistent sound source: the sound is incorrectly and compellingly localized to the site of the visual object (Jackson, 1953; Thurlow and Rosenthal, 1976; Warren et al., 1982). Unlike the previous examples, visual capture is immediate, and its effect persists only as long as a subject views an appropriate target while localizing sound.

These kinds of observations have led to the widely accepted view that, with regard to spatial localization, vision dominates audition. However, the initial conditions in these experiments are either that auditory localization is inaccurate (perturbation of auditory cues) or that both auditory and visual localization are accurate but are mutually inconsistent (visual capture). The ultimate test of visual dominance would be a situation in which visual-auditory discordance is caused by visual localization that is inaccurate (i.e., the perceived location of an object is not its actual location) while auditory localization is intrinsically correct. Such a situation is created by mounting binocular displacing prisms over the eyes: the prisms shift the visual field without interfering with auditory spatial cues. Humans subjected to these conditions respond by altering the perceived zero positions of the eyes so as to compensate for the prismatic displacement. This reestablishes both visual localization accuracy and visualauditory concordance (Harris, 1965, 1980; Lackner, 1973, 1981). However, barn owls do not have this option because the eyes are essentially immobile in the head. Thus, barn owls offer the opportunity to put the principle of visual dominance over sound localization to this ultimate test.

To maximize the potential for effecting change, we mounted displacing prisms on baby owls. The prisms were placed as the eyelids first began to open, when the ocular medium is still cloudy and before the eyes assume their adult orientations (Knudsen, 1989). At this stage, the ear canals are no more than pinholes, and the facial ruff (the acoustically reflective surface of the external ear that is primarily responsible for creating localization cues) does not yet exist (Knudsen et al., 1984a). The owls wore the prisms continuously for months. When tested as juveniles, they had altered sound localization (within limits) to match visual localization, even though visual localization was incorrect (Knudsen and Knudsen, 1989a).

\section{Materials and Methods}

Prisms. Matched binocular Fresnel prisms (Vision Care/3M) were mounted on 12 baby barn owls (Tyto alba) that were 10 to $15 \mathrm{~d}$ old (Table 1). One of 3 prism strengths was used: 20 diopter $\left(11^{\circ}\right.$ displacement, $n=2)$, 40 diopter ( $23^{\circ}$ displacement, $\left.n=4\right)$, or 60 diopter $\left(34^{\circ}\right.$ displacement, $n=6$ ). The prisms were oriented to displace the visual freld to the right and were mutually aligned such that a collimated beam 
passed through either lens diffracted identically. Blinders on the sides of the frames blocked peripheral vision. The prisms were held in place with lightweight aluminum frames that were strapped to the head until the owls were about $30 \mathrm{~d}$ old and then, once the skull had hardened, were bolted to the skull (Fig. 1). The bolts were cemented to the skull while the owls were anesthetized with halothane and nitrous oxide.

Experience. The baby owls were raised in 3 communal cages containing 2,3 , and 7 birds, respectively. They interacted vigorously, vocalized frequently, and used vocalizations and vision to locate siblings. As they grew, they actively explored their cages by pecking, scratching, and pouncing on objects. As a result, they were exposed to a great deal of temporally correlated visual and auditory stimulation. When they were about $50 \mathrm{~d}$ old, they were placed in individual cages so that food intake could be controlled. Training began at this age for most of the owls. Throughout the training and testing period, they were handled almost every day and were occasionally released in a large flight room for additional experience and exercise.

Test paradigm. Sound localization was quantified by measuring the orientation of the head relative to acoustic targets. The companion study (Knudsen and Knudsen, 1989a) demonstrated that the orientation of the head is a reliable indicator of the owl's perception of stimulus source location.

Behavioral tests were conducted with the owl perched in a darkened sound isolation chamber (IAC 404A). The owl was monitored using infrared illumination and a videocamera. Auditory stimuli were generated by a movable $4-\mathrm{cm}$ loudspeaker and consisted of repetitive noise bursts of variable amplitude, duration, and repetition rate. Visual stimuli consisted of a glow from a light-emitting diode that was centered in the speaker cone. The speaker and photodiode were attached to a semicircular track that moved by remote control (Fig. 2). Movements of the track along its axis altered the elevation of the stimuli with respect to the owl, and rotation of the track around a vertical axis changed the azimuth of the stimuli.

At the beginning of a test session, the owl was placed in the sound chamber and the lights were extinguished. The speaker-diode was moved to a random location that was within $25^{\circ}$ of the owl's visual axes (crosses in Fig. 3). Then, either the auditory or the visual stimulus was presented for up to $2 \mathrm{sec}$, which was long enough to allow the owl to make several sequential head movements to maximize its accuracy. If the animal did not respond, the stimulus was turned off and a waiting period of about $30 \mathrm{sec}$ ensued. Typically, however, the animal responded immediately with one quick head movement and a sustained fixation. The coordinates of the final head orientation were recorded, a reward light was

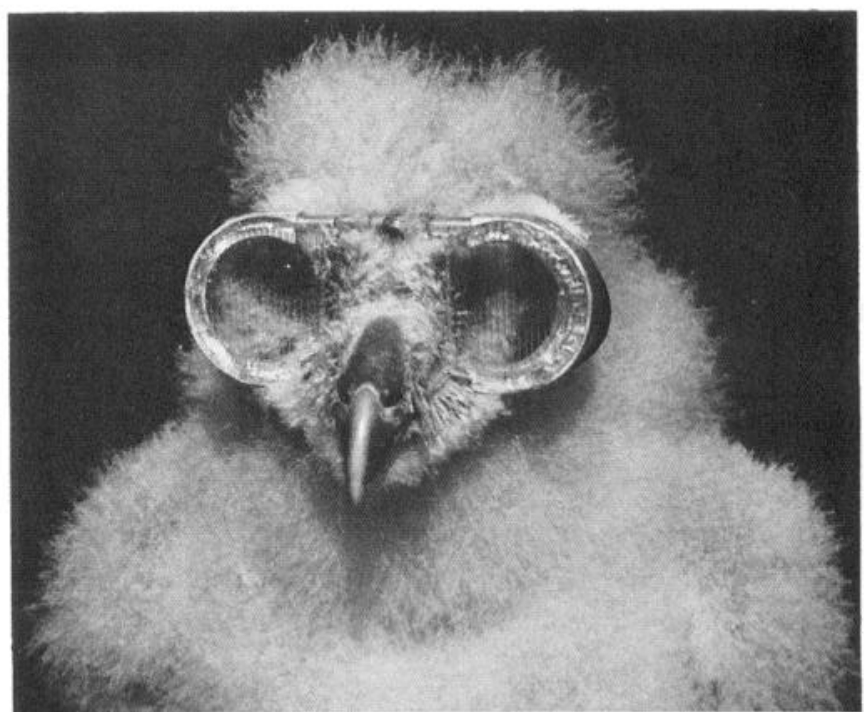

Figure 1. A baby barn owl, $28 \mathrm{~d}$ old, wearing binocular Fresnel prisms that displaced the visual field $34^{\circ}$ to the right. Photograph indicates the optical quality of the prisms and the large visual field afforded by the spectacles. The vertical lines on the prisms are the edges of the individual prism elements that allow large optical displacements to be achieved with thin prisms.

activated, and a food reward was delivered. The reward criteria were that the owl turn its head crisply and fixate on a point during the stimulus. The reward was not contingent on the accuracy of the response. This was done because we could not know a priori how prism-reared birds would orient to the stimuli. After every trial, the speaker-diode was moved to a new, random location.

Since the test trials were carried out in the dark and with only the auditory or the visual stimulus active during any one trial, auditory localization was independent of visual localization, and vice versa. The tests themselves were not used to shape the animal's response to one or the other stimulus. Thus, measured changes in sound localization

Table 1. Effect of prism experience on sound localization

\begin{tabular}{|c|c|c|c|c|c|c|}
\hline \multicolumn{2}{|l|}{ Owl } & \multirow[t]{2}{*}{$\begin{array}{l}\text { Prism } \\
\text { strength } \\
\text { (degree } \\
\text { right) }\end{array}$} & \multirow[t]{2}{*}{$\begin{array}{l}\text { Prisms } \\
\text { on } \\
\text { (age in } \\
\text { days) }\end{array}$} & \multirow[t]{2}{*}{$\begin{array}{l}\text { Prisms } \\
\text { off } \\
\text { (age in } \\
\text { days) }\end{array}$} & \multirow[t]{2}{*}{$\begin{array}{l}\text { Prisms on }{ }^{a} \\
\text { Aud-Vis difference } \\
\text { (deg) } \pm \\
\text { auditory SD } \\
\text { (deg) }\end{array}$} & \multirow[t]{2}{*}{$\begin{array}{l}\text { Prisms off } \\
\text { Aud-Vis difference } \\
\text { (deg) } \pm \\
\text { auditory SD } \\
\text { (deg) }\end{array}$} \\
\hline (number) & (identity) & & & & & \\
\hline 1 & $\mathrm{Be}$ & 11 & 10 & 102 & $\mathrm{~L} 0.2 \pm 2.1$ & R $7.5 \pm 3.1$ \\
\hline 2 & $\mathrm{Ma}$ & 11 & 12 & 103 & $\mathrm{~L} 1.6 \pm 3.0$ & R $9.4 \pm 2.0$ \\
\hline 3 & Is & 23 & 10 & 229 & $\mathrm{~L} 1.1 \pm 3.6$ & $R 16.0 \pm 5.8$ \\
\hline 4 & Ro & 23 & 14 & 79 & $\mathrm{~L} 1.1 \pm 2.6$ & R $17.4 \pm 1.8$ \\
\hline 5 & $\mathrm{Ol}$ & 23 & 10 & - & $\mathrm{R} 0.1 \pm 3.1$ & - \\
\hline 6 & $\mathrm{Da}$ & 23 & 15 & 98 & $\mathrm{R} 0.1 \pm 1.7$ & R $20.9 \pm 1.9$ \\
\hline 7 & $\mathrm{Fi}$ & 34 & 12 & 159 & L $12.3 \pm 4.6$ & R $17.6 \pm 3.4$ \\
\hline 8 & $\mathrm{Mi}$ & 34 & 12 & 214 & L $11.6 \pm 2.0$ & R $17.1 \pm 2.1$ \\
\hline 9 & Wa & 34 & 12 & 111 & L $14.8 \pm 3.0$ & R $12.4 \pm 1.6$ \\
\hline 10 & $\mathrm{Bl}$ & 34 & 15 & 79 & L $15.8 \pm 4.1$ & R $10.9 \pm 2.6$ \\
\hline 11 & Do & 34 & 12 & 87 & L $11.4 \pm 2.6$ & R $10.8 \pm 3.4$ \\
\hline 12 & Ds & 34 & 14 & 90 & L $11.9 \pm 2.1$ & R $14.8 \pm 3.0$ \\
\hline
\end{tabular}

Other data from some of these owls appear in other reports. The number of the owl is adapted for each report so that the order represents a logical progression for that study. The identity of the owl is maintained across studies to enable comparison of data from the same animal presented in different reports.

"Final measurement before prisms were removed.

Aud-Vis difference is the difference between the mean orientation to the auditory stimulus and the mean orientation to the visual stimulus.

First measurement after prism removal. 


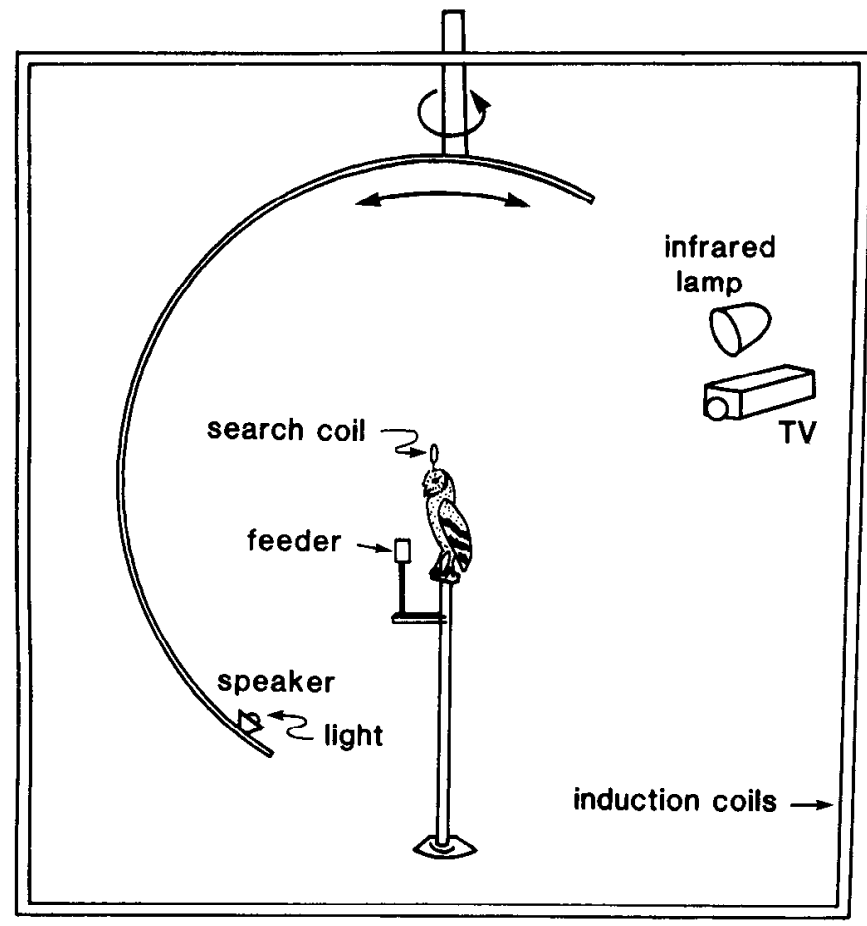

Figure 2. The experimental set-up. This equipment was housed in a sound-isolation chamber. Induction coils generated mutually orthogonal magnetic fields at the site of the search coil. The currents induced in the search coil depended on the orientation of the coil (and, therefore, of the head) relative to the magnetic fields. Auditory and visual stimuli were delivered from a remotely controlled, movable loudspeaker and light-emitting diode, respectively. A crisp response to a stimulus was rewarded with food from the feeder. The animal was watched with an infrared video system; otherwise, the room was dark.

accuracy were caused by experience gained outside the experimental chamber.

Measurement of head orientation. Head orientation was monitored using a search coil system (C-N-C Engineering). Pairs of induction coils, each $1.8 \mathrm{~m}$ in diameter, surrounded the speaker-diode moving system inside the sound isolation chamber (Fig. 2). The coils generated magnetic fields that were mutually orthogonal at the center of the chamber. The owl's perch was positioned so that the head was at the center of the chamber. A search coil was attached to a socket that fit into a clip cemented to the skull; the clip was attached while the animal was anesthetized with halothane and nitrous oxide. Currents induced in the search coil depended on the orientation of the search coil and, therefore, on the orientation of the head relative to the magnetic fields. These signals were calibrated before each test session and were interpreted online. The precision of the system was about $\pm 0.1^{\circ}$ in the frontal area sampled most heavily (angles lcss than $30^{\circ}$ from $0^{\circ}$ azimuth, $0^{\circ}$ elevation) and decreased to about $\pm 1.0^{\circ}$ at the most extreme positions.

Accuracy and precision of sound localization. For each response, the position of the stimulus source was subtracted from the search coil reading to yield coil orientation relative to stimulus source location. A test session consisted of 10 to 25 responses to the auditory stimulus and a similar number of responses to the visual stimulus. Mean and SD were calculated separately for the auditory and visual responses. The difference between these mean values is reported as auditory-visual (Aud-Vis) differences. The SD of the responses to the auditory stimulus is reported as the precision of sound localization. The Aud-Vis difference was a measure of sound localization relative to visual localization that could be compared from day to day.

In some cases, it is clearer to present the data as mean head orientation relative to source location. Calculation of mean hcad oricntation rcquired knowledge about the orientation of the search coil relative to the head ("coil error"). Coil error was measured as the mean search coil reading for all orientations to visual stimuli with the prisms removed. This assumes that prism-reared owls orient the head directly at a light source once the prisms arc removed. This assumption was validated for several birds by photographing the face of the owl as it oriented to a visual stimulus immediately after prism removal. Photographs were taken with the camera lens positioned just below the photodiode aimed at the head. To assess the precision of this technique for judging head orientation, additional photographs were taken with the camera lens centered $2^{\circ}$ to the left of the photodiode and $2^{\circ}$ to the right of the photodiode. These photographs demonstrated that, in every case, the head oriented toward the visual target $\left( \pm 1^{\circ}\right)$ immediately after prism removal. These data imply that eye position is unaffected by prismrearing, an assumption confirmed by direct retinoscopic measurements in these birds (Knudsen, 1989). Search coil error was computed for each bird after the final test session was completed. Mean head orientation relative to the stimulus source, which we define as localization accuracy (Knudsen and Knudsen, 1989b), was derived by subtracting the coil error from the mean auditory and mean visual response for each day.

Optical properties of the prisms. The plastic Fresnel prisms used in this study are made for treating strabismic humans. A human observer notices a slight degradation of resolution throughout the visual field; however, a barn owl, because of its poor acuity (Bravo and Pettigrew, 1981), may not detect this blur. Each prism was $18 \mathrm{~mm}$ wide and 23 $\mathrm{mm}$ high and was oriented orthogonal to the visual axis. The extent of the visual field afforded by the prisms was measured in an anesthetized owl $(5.0 \mathrm{mg} / \mathrm{hr}$ ketamine and $0.3 \mathrm{mg}$ diazepam injected into the breast muscle). The head was centered in a perimetry system that enabled angular measurements accurate to $\pm 0.5^{\circ}$ (Knudsen, 1989). The visual axes of the eyes were aligned with $0^{\circ}$ elevation and symmetrically with respect to $0^{\circ}$ azimuth by sighting retinal landmarks. Thus, elevation corresponds to angles above $(+)$ or below $(-)$ the owl's visual plane, and azimuth corresponds to angles right $(R)$ or left $(L)$ of the owl's midsagittal plane, both angles measured from the center of the head. The region of space from which the retina could be seen (through the prisms) was defined as the extent of the visual field for each eye.

The visual field decreased with increasing prism strength (Fig. 3). Normally, the visual field of each eye extends about $125^{\circ}$ in azimuth. The $11^{\circ}$ prisms decreased this to $70^{\circ}$ for the right eye and to $100^{\circ}$ for the left eye. The $34^{\circ}$ prisms decreased it further to $60^{\circ}$ for the right eye and to $80^{\circ}$ for the left eye. In each case, the visual field was limited by the midsagittal lore feathers on the nasal side and by the optical effects of the prisms on the temporal side. The extent of the binocular visual field was affected little by any of the prisms.

Acoustical effects of the prisms. Centering the prisms over the eyes required that the prisms be in front of the ear canal openings, which are located behind and lateral to the eyes. Alterations of acoustic cues caused by the presence of the prisms could affect sound localization accuracy and precision. We measured the effect of the prisms on sound entering the ear canals in an anesthetized owl $(5.0 \mathrm{mg} / \mathrm{hr}$ ketamine, 0.3 $\mathrm{mg}$ diazepam) and used this information to limit the region of space from which sounds were presented in our experiments.

The head of the anesthetized owl was aligned and fixed at the center of a speaker moving system as described by Knudsen (1982). A calibrated probe tube $(1.5 \mathrm{~mm}$ internal diameter, $150 \mathrm{~mm}$ long) attached to a half-inch Bruel and Kjaer microphone (4165) was inserted into each ear canal and positioned in front of the eardrum. Amplitude and phase spectra in each ear and the transfer functions between the ears produced by a wideband noise source from various locations were measured (Nicolet $660 \mathrm{~A}$ spectrum analyzer) before and after mounting the prisms.

For frequencies below $5 \mathrm{kHz}$, the prisms had no effect on the amplitude or timing of sound in either ear for any source location in the frontal hemifield. For frequencies above $5 \mathrm{kHz}$, the prisms attenuated the amplitude in either ear by less than $3 \mathrm{~dB}$ for source locations between $0^{\circ}$ and $-15^{\circ}$ elevation at any azimuth. Amplitudes were attenuated in the left ear by 0 to $10 \mathrm{~dB}$ for sources located between $0^{\circ}$ and $+25^{\circ}$ at any azimuth. Amplitudes were attenuated in the right ear by 0 to $8 \mathrm{~dB}$ for sources located between $-15^{\circ}$ and $-25^{\circ}$ at any azimuth. The prisms altered timing by $10 \mu \mathrm{sec}$ or less within the elevations of $+25^{\circ}$ to $-25^{\circ}$ at any azimuth. Above or below this area, large frequency-dependent effects on both amplitude and timing were observed.

The effect of prism rearing on sound localization that is described in this report is a change in azimuthal accuracy. Barn owls localize in azimuth primarily on the basis of interaural timing cues (Moiseff and Konishi, 1981), which were essentially unaffected by the presence of the prisms. The acoustical effects of the prisms on sound amplitude could have affected localization in elevation, but elevational effects are largely 

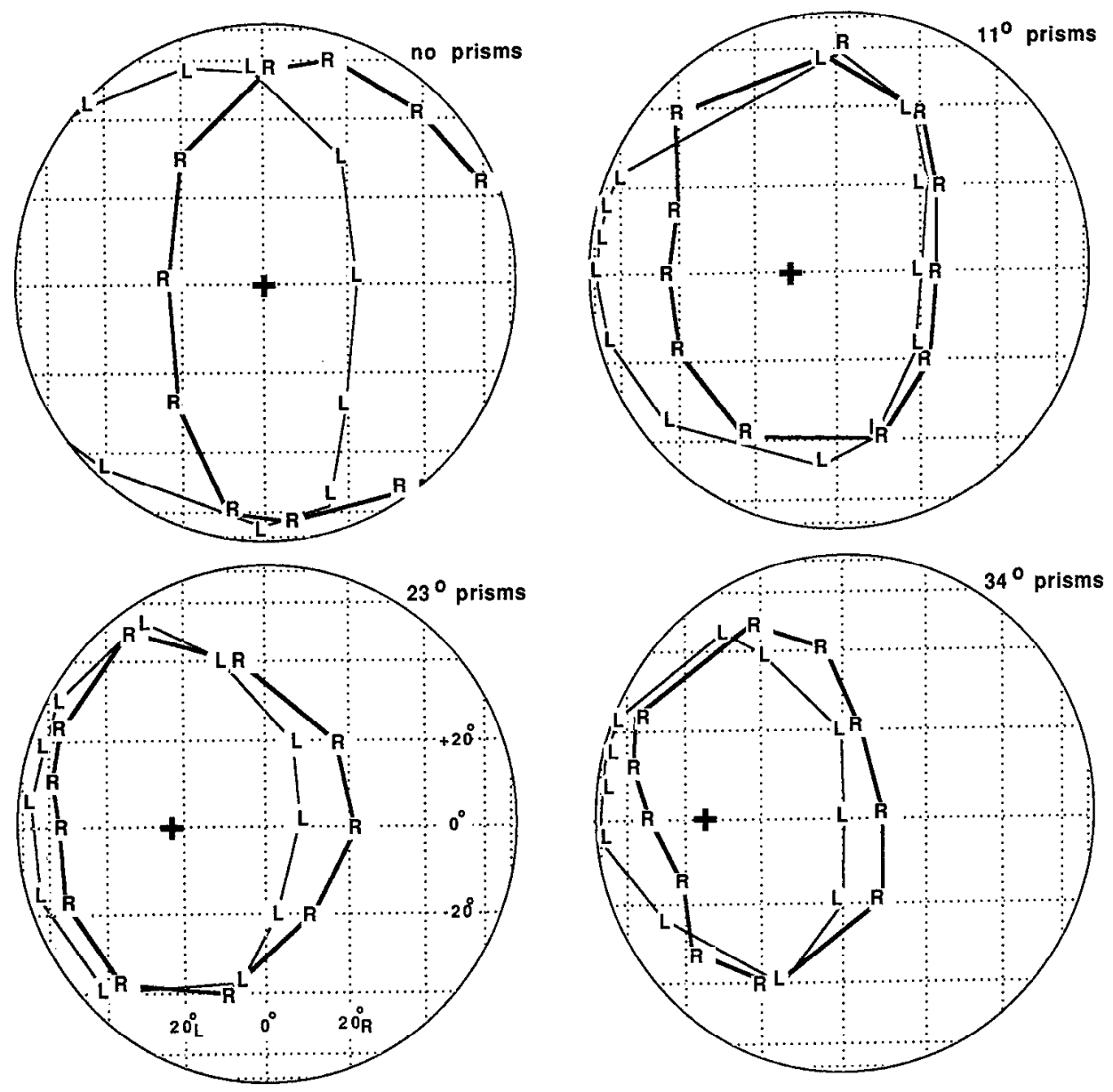

Figure 3. Visual fields afforded by the prism spectacles. The visual field of the right $(R)$ and left $(L)$ eyes were measured ophthalmoscopically as that region of space from which the retina could be sighted. The borders of the visual fields are plotted on globes representing space in degrees left or right of the owl's midsagittal plane (azimuth) and in degrees above or below the owl's visual plane (elevation). These data are from a single barn owl measured without prisms and while wearing each of the three strengths of prisms, as indicated. Crosses indicate the projections of the visual axes.

ignored in this study. Moreover, by continuing the stimulus until the owl settled on a particular orientation, the owl's final judgment was based on a source located almost directly in front of its face, a region from which auditory localization cues were unaffected by the prisms. The accuracy of the responses to sound sources before and immediately after prism removal (as shown in Fig. 4) demonstrates that the presence of the prisms did not alter sound localization as measured in this study.

\section{Results}

\section{Visual displacement shifts sound localization}

The effect of prism rearing on the development of sound localization accuracy was first tested by raising 2 owls with binocular, $11^{\circ}$ right-displacing prisms (owls 1 and 2; Table 1 ). The prisms were mounted over the eyes just as the eyelids first opened. We began training the owls when they were $80 \mathrm{~d}$ old, and we gathered the first complete sets of data when they were 94 and $96 \mathrm{~d}$ old, respectively.

Unlike normal owls, these owls did not orient the head directly at auditory and visual stimuli. In response to either a sound or a light, the head oriented to the right of the stimulus source by an amount approximately equal to the prismatic displacement (Fig. $4 A$ ). This behavior aimed the prismatically displaced projections of the visual axes of the eyes at the auditory and visual targets. Aud-Vis differences (see Materials and Methods) ranged from $\mathrm{L} 0.6^{\circ}$ to $\mathrm{R} 0.2^{\circ}$ for owl 1 and from $\mathrm{L} 1.6^{\circ}$ to R0.3 for owl 2 .

When the prisms were removed at 102 and $103 \mathrm{~d}$ old, respectively, the owls oriented the head directly at the visual stimulus. However, when presented with an auditory stimulus, they continued to orient to the right of the source, owl 1 by $7.5^{\circ}$ \pm 3.1 and owl 2 by $9.4^{\circ} \pm 2.0$ (Table 1 , Fig. $4 A$ ). When auditory and visual stimuli were presented simultaneously from the same location, the orientation of the head alternated from one position, directed at the target (visual localization), to another, directed to the right of the target (auditory localization). Thus, surprisingly, the owls did not exhibit visual capture under these conditions. These sound localization errors disappeared slowly over a period of weeks after prism removal (Fig. $5 A$ ).

\section{Limited shift of sound localization}

To determine whether there is a limit to the shift in sound localization that prism rearing can induce, we raised other owls with stronger prisms, $23^{\circ}$ or $34^{\circ}$ right-displacements (Table 1 ). All owls raised with $23^{\circ}$ prisms $(n=4)$ had shifted sound localization to match visual localization by the time they were first tested at 62 to $66 \mathrm{~d}$ old. With the prisms on, the head oriented far to the right both of auditory and of visual stimuli (Figs. $4 B$ and 6); Aud-Vis differences were small, ranging from L $1.1^{\circ}$ to $\mathrm{R} 0.1^{\circ}$ for 4 birds (Table 1 ). After removal of the prisms, the head oriented directly at the visual stimulus (documented photographically) but continued to orient $16.0^{\circ}$ to $20.9^{\circ}$ to the right of the auditory stimulus (Table 1, Fig. 4). These auditory localization errors disappeared gradually over periods of weeks or months (Fig. 5).

In contrast to the owls reared with weaker prisms, those reared with $34^{\circ}$ prisms $(n=6)$ oriented to auditory stimuli differently than they did to visual stimuli while the prisms were still on: 


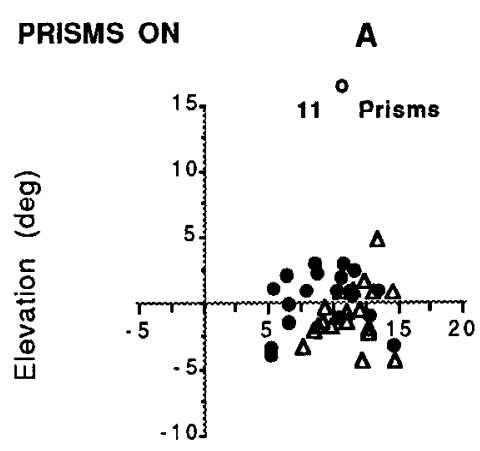

PRISMS OFF

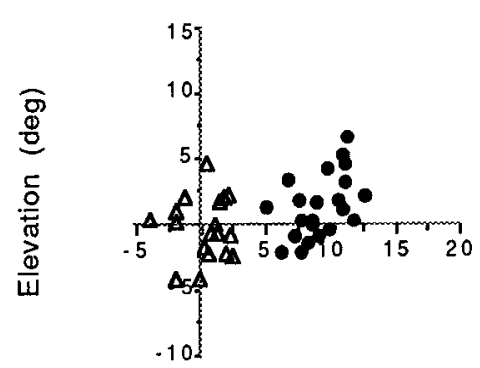

B
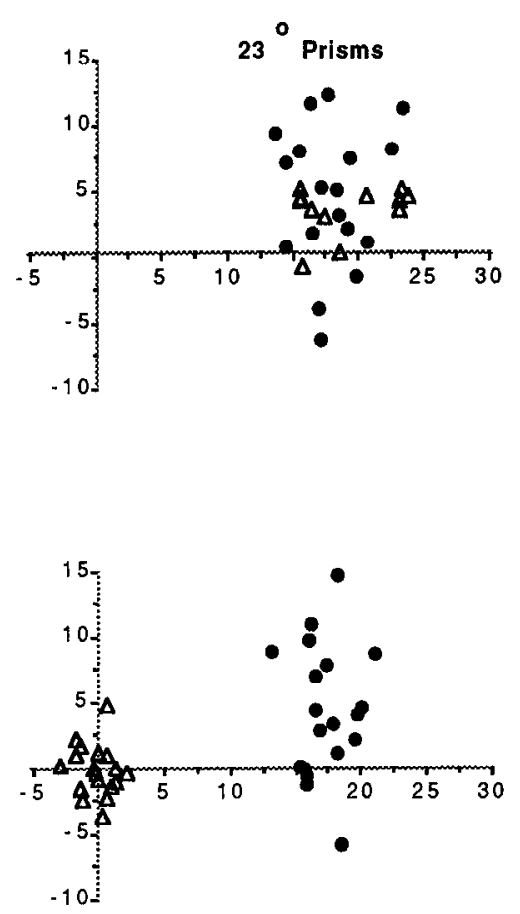

Azimuth (deg)
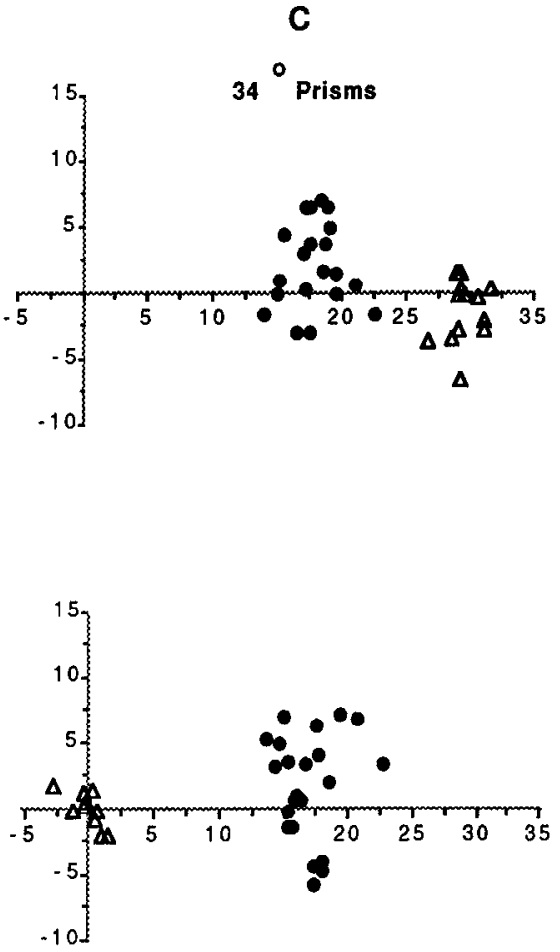

Figure 4. Individual responses to auditory and to visual stimuli with prisms on and off. The data are from owl $2(A)$, owl $4(B)$, and owl $8(C)$. Each symbol represents a single response relative to the position of the stimulus source. The position of the stimulus is the origin of this coordinate system. Closed circles represent responses to the sound source; open triangles represent responses to the light source. Each plot shows data collected in a single test session on the day before prism removal (top) or on the first day with the prisms off (bottom). Only visual responses changed immediately after prism removal. The prisms were removed on day 103 for owl 2, on day 79 for owl 4, and on day 214 for owl 8 .

Aud-Vis differences ranged from L8. $6^{\circ}$ to L15.8 (Figs. 4-6). Clearly, the adjustment of sound localization was incomplete in these owls. To determine whether this was due to insufficient time for adjustment, the prisms were left on and sound localization was followed for an additional 100 and $130 \mathrm{~d}$ in owls 7 and 8 , respectively. Over this period of time, Aud-Vis differences ranged from $\mathrm{L} 8.6^{\circ}$ to $\mathrm{L} 13.4^{\circ}$ for owl 7 (Fig. 5) and from L9. $4^{\circ}$ to $\mathrm{L} 13.1^{\circ}$ for owl 8 , but neither owl showed any indication of further systematic adjustment of sound localization. These data demonstrate (1) that the adjustment of sound localization had stopped before the age at which the owls were first tested ( $60 \mathrm{~d}$ old) and (2) that the lack of complete adjustment was not due to inadequate time for adjustment.

Following prism removal, these owls oriented the head directly at the visual target but $10.8^{\circ}$ to $17.6^{\circ}$ to the right of the auditory target (Fig. 4C). These sound localization errors were more variable (across birds) than those induced in the other birds by weaker prisms, and the errors of 3 of these owls were even smaller than any of the errors induced in the owls reared with $23^{\circ}$ prisms (Table 1, Fig. 6). As described for the other owls, these sound localization crrors disappeared over a period of weeks or months following prism removal (Fig. 5).

\section{Prism effects on localization precision}

The azimuthal precision (SD) of sound localization was poorer for some of the prism-reared owls than it was for normal owls measured in a previous study (Knudsen et al., 1984a). The average azimuthal precision of normal owls is $1.8^{\circ} \pm 0.6(n-$ 22). In this study, owls $3,5,7$, and 10 oriented to auditory stimuli with SDs that ranged from $3.1^{\circ}$ to $5.7^{\circ}$. An $F$ test $(p<$ 0.01 ) indicated that the variance of the data from each of these birds was larger than the variance of comparable data from normal owls. However, all of these birds were orienting to sounds with normal precision within $25 \mathrm{~d}$ of prism removal.

\section{Prism effects on visual orientation}

The immediate effect of prism removal was to change the orientation of the head to visual targets. Orientations to auditory targets remained unchanged (Fig. 4). However, the magnitude of the shift in the mean visual response before and after prism removal was always smaller (except in owl 2) than that expected from the optical shift caused by the prisms, $7.7^{\circ}$ to $11.0^{\circ}$ in owls 1 and $2\left(11^{\circ}\right.$ prisms $), 17.1^{\circ}$ to $20.8^{\circ}$ in owls 3,4 , and $6\left(23^{\circ}\right.$ prisms), and $22.2^{\circ}$ to $29.9^{\circ}$ in owls 7 to 12 ( $34^{\circ}$ prisms). Figure 6 documents the discrepancies between theoretical and observed shifts in visual orientations as the Aud-Vis differences measured before and after prism removal compared with the strength of the respective prisms.

\section{Discussion}

\section{Comparison with visual capture}

The adjustment of sound localization caused by prism rearing is superficially similar to the phenomenon of visual capture. In both cases, the brain resolves a discrepancy in spatial information provided by the auditory and visual systems by altering sound localization to match visual localization (Shelton and Searle, 1980; Warren et al., 1982). Also, in both cases there is a limit to the angular discrepancy that can be overcome: ap- 
A
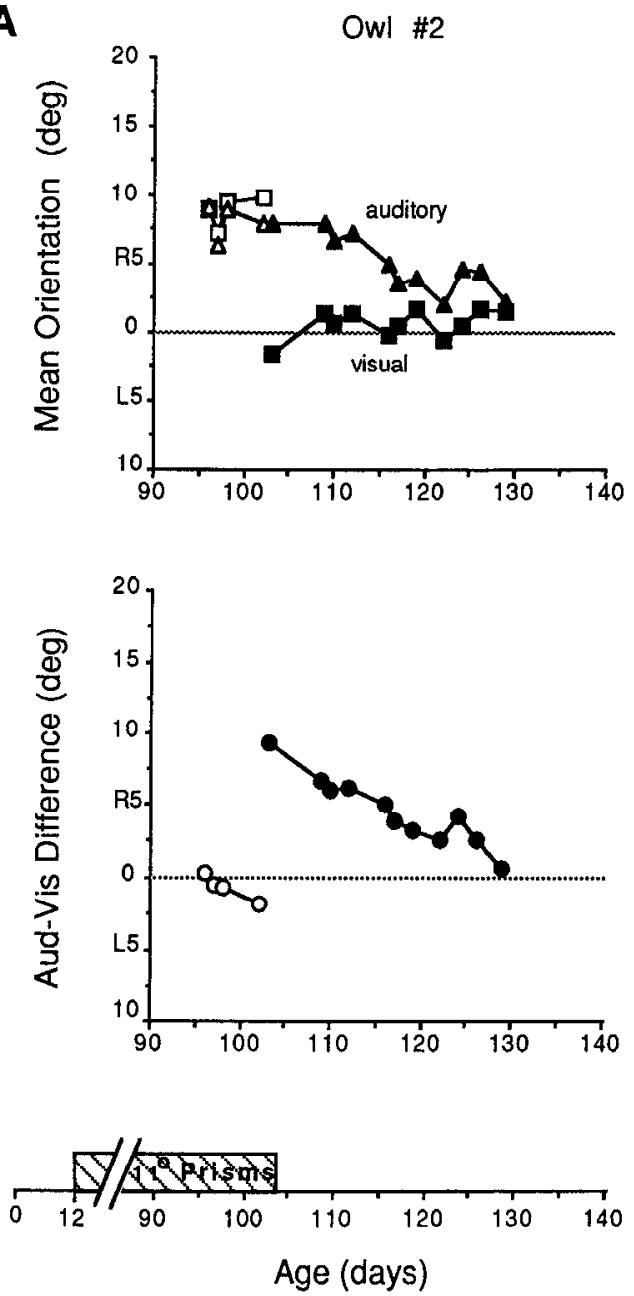

B
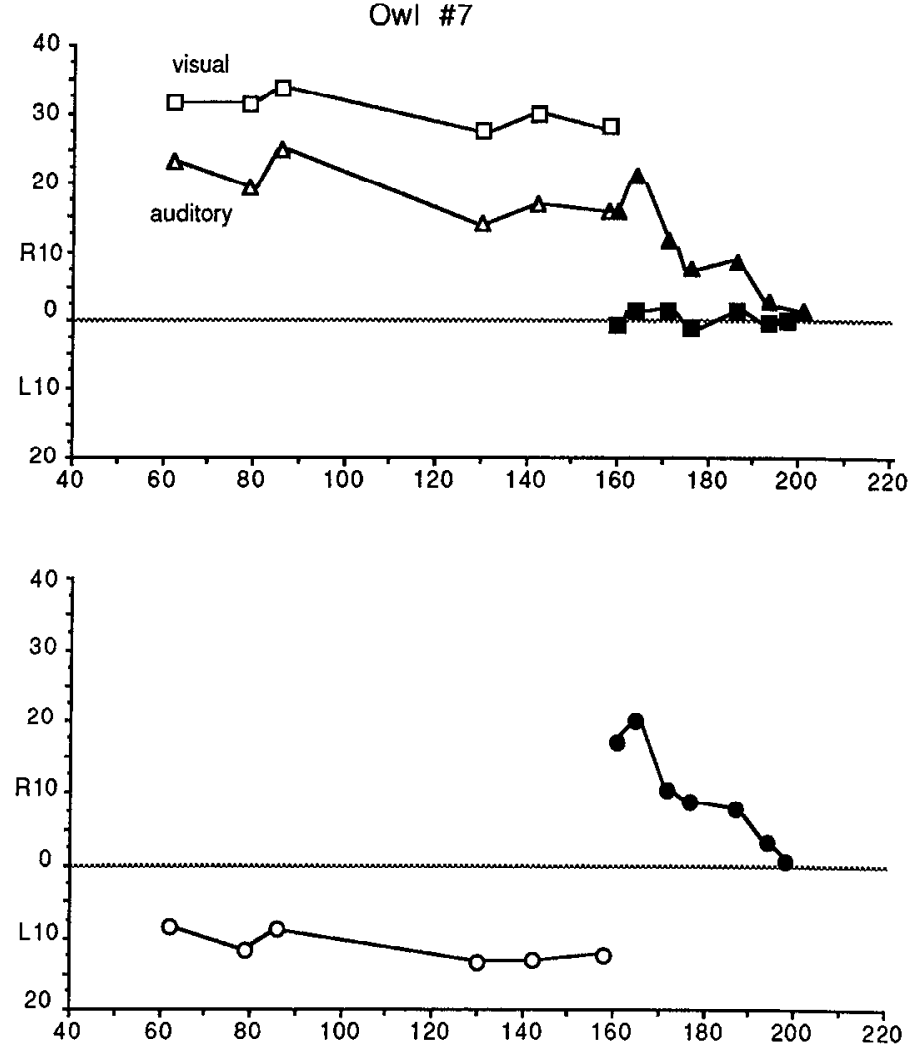

Figure 5. Auditory and visual localization by owls 2 and 7 measured during and after exposure to right-displacing prisms. $A$, Mean orientation relative to a sound source (triangles) and relative to a light source (squares) are plotted as functions of age in days with the prisms on (open symbols) and off (closed symbols). Mean orientation was calculated from 12 to 23 responses to each stimulus. $B$, Mean visual orientation has been subtracted from mean auditory orientation to yield the auditory-visual (Aud-Vis) difference. This value indicates auditory localization relative to visual localization. Bars across the bottom represent the histories of the owls.

proximately $20^{\circ}$ for prism rearing in barn owls, and approximately $30^{\circ}$ for visual capture in humans (Jackson, 1953; Thurlow and Rosenthal, 1976). However, these 2 phenomena are essentially different. Visual capture causes a change in sound localization that is immediate and requires the simultaneous observation of an appropriate visual target; when the visual target disappears, sound localization reverts to normal. Thus, visual capture represents a visual override of sound localization. In contrast, prism rearing causes changes in sound localization that probably occur gradually (based on the gradual recovery of accuracy following prism removal; Fig. 5) and do not require simultaneous visual stimuli to be maintained. Moreover, the capacity for prism-induced adjustment decreases dramatically with age in barn owls (Knudsen and Knudsen, 1989b). These properties suggest that the effect of prism rearing is not a visual override of sound localization but a recalibration of auditory cues at a more fundamental level.

\section{Visual dominance over sound localization}

Owls raised wearing displacing prisms calibrate their interpretation of auditory cues so that sound localization is consistent with visual localization even though experience demonstrates that visual spatial information is incorrect. Owls raised from the day of eyelid opening viewing the world through displacing prisms continue to commit systematic errors when attempting to fly toward or strike at visual targets (Knudsen and Knudsen, 1989a). Thus, the dominance of vision over sound localization does not depend on the demonstrated validity of visual localization; apparently, visual dominance is innate.

In the prism-reared owls, the validity of normal auditory cuelocation associations could have been confirmed by many nonvisual cues, such as the success of strikes at sound sources, the pattern of change of auditory cues with head movement relative to a sound source, and the correspondence of tactile and auditory spatial information. Vision overrode these other potential cues and induced inaccurate sound localization. Thus, concordance of auditory with visual space carries greater weight in the calibration process than does any other source of verifying information.

\section{Auditory plasticity is limited}

Despite the dominance of vision, its ability to alter sound localization is limited. Owls raised with $34^{\circ}$ optical displacements never oriented in the same way to auditory and to visual stimuli, 


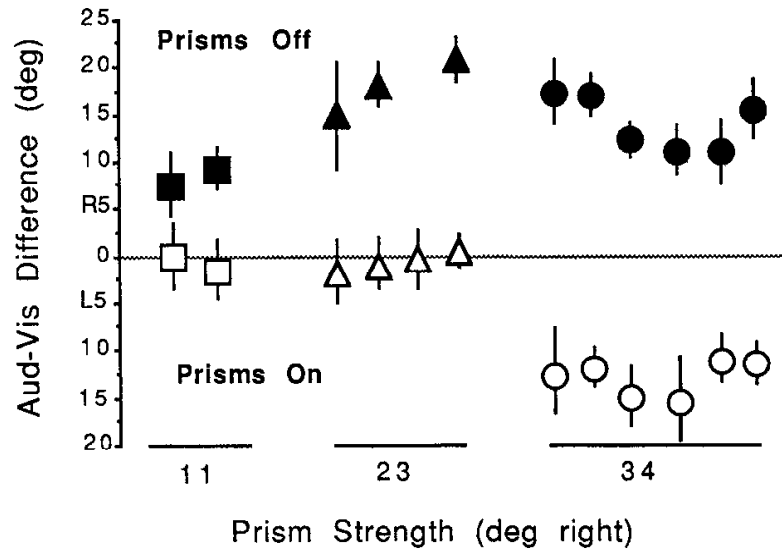

Figure 6. Summary of the effect of prism rearing on sound localization. Each symbol represents the Aud-Vis difference of an owl measured on the day before (open symbols) or on the day after (closed symbols) prism removal. The data are ordered along the abscissa according to owl number and are grouped by prism strength. Error bars indicate the SDs of the azimuthal component of sound localizations. The data indicate that adjustment of sound localization to $11^{\circ}$ and $23^{\circ}$ displacements was relatively complete, whereas adjustment to $34^{\circ}$ displacements was only partial.

although they experienced an auditory-visual spatial discordance for periods of up to 7 months (Table 1). Moreover, following prism removal, the magnitudes of the sound localization error (R $10^{\circ}$ to $\mathrm{R} 17.6^{\circ}$ ) were considerably less than the optical displacement the owls had experienced. Clearly, in these birds, the adjustment of sound localization was incomplete (Fig. 6).

Lack of complete adjustment could have been due to optical properties peculiar to the $34^{\circ}$ prisms or to biological properties of the adjustment mechanism. We cannot discount entirely the importance of peculiar optical properties. However, all evidence argues against this as an explanation. First, the optical quality of the $34^{\circ}$ prisms was as good as that of the other prisms. Human observers could detect little difference between the $34^{\circ}$ prisms and the $23^{\circ}$ prisms when viewing a uniformly textured screen that obscured the magnitude of the displacements. Moreover, the birds that wore these prisms developed normal eye alignment, which requires fused binocular form vision (Knudsen, 1989), and they were able to see small targets at a distance and to use vision to guide their approach and strike (Knudsen and Knudsen, 1989a). Second, the extent of the visual field afforded by $34^{\circ}$ prisms was only slightly less than that afforded by the $23^{\circ}$ prisms (Fig. 3): the edge of the field of each eye was located about $10^{\circ}$ further to the left with the $34^{\circ}$ prisms. The extent of the binocular field through the $34^{\circ}$ prisms was almost normal. Third, all of the birds reared with $34^{\circ}$ prisms did, in fact, adjust sound localization by a considerable amount: sound localization errors measured with the prisms removed ranged from L $11.4^{\circ}$ to L15.8 ${ }^{\circ}$ (Fig. 6).

What prevented the birds wearing $34^{\circ}$ prisms from completing the adjustment of sound localization and achieving auditoryvisual concordance? We propose that incomplete adjustment in this case reflects a limitation in the neural mechanisms that underlie adjustment. Logically, the limit could reside in the circuitry that undergoes change, in the mechanism that instructs change, or in both. If the restricted range of adjustment were due only to a limitation in the association circuitry itself, then sound localization errors induced by a large optical displacement should asymptote at some limiting value. This predicts that the localization errors of owls raised with $34^{\circ}$ prisms should be as large as or slightly larger than those of owls raised with $23^{\circ}$ prisms. The data in Figure 6 show that the sound localization errors induced by $34^{\circ}$ prisms were certainly no larger than those induced by $23^{\circ}$ prisms. If anything, the errors tended to be smaller. However, the significance of this tendency cannot be assessed because of the small sample size.

Another possibility is that the limited range of adjustment is due to a property of the mechanism that induces change. For example, the strength of the instructional force might increase with the size of the initial auditory-visual discordance but reach a limit, or decrease, when the initial discordance exceeds $20^{\circ}$. If this is the case, the strength of the instructional force must depend specifically on initial, and not on current, discordance because, otherwise, adjustments would have continued to completion.

Whatever the cause of the limit might be, the existence of a limit to the adjustment of sound localization implies that the brain possesses an innate expectation of auditory cue-location correspondences. The cue-location associations that underlie sound localization can be altered only so far from these innate expectations.

In summary, the effect of prism rearing on sound localization is consistent with the following model. Early in development, neural circuitry is established by innate mechanisms that associate at least basic auditory cue values with anticipated, corresponding locations (e.g., $0 \mu \mathrm{sec}$ interaural time difference and $0 \mathrm{~dB}$ interaural intensity difference with straight ahead). Built into this circuitry is a degree of flexibility that permits fine-tuning by experience. The connectivity of the association circuitry is fine-tuned by an instructional force that results from a comparison of auditory and visual spatial information. An auditoryvisual mismatch results in an adjustment in auditory cue-location associations. Normally, experience confirms the innate auditory associations, and the instructional mechanism simply refines the circuitry according to idiosyncracies in the cue-location correspondences experienced by the individual (due to the exact shape and size of the head and ears). Because the range of adjustment required normally is small, the capacity for arbitrarily large adjustment has not evolved. A $20^{\circ}$ adjustment appears to represent the largest change (at least in the azimuthal dimension) away from innate associations that the instructional mechanism can induce. Larger spatial mismatches generate an instructional force, but the force is inadequate (because of limits in the association circuitry, in the instructional mechanism, or both) to achieve complete auditory-visual concordance.

\section{References}

Bravo, H., and J. D. Pettigrew (1981) The distribution of neurons projecting from the retina and visual cortex to the thalamus and tectum opticum of the barn owl, Tyto alba, and the burrowing owl, Speotyto cunicularia. J. Comp. Neurol. 199: 419-441.

Harris, C. S. (1965) Perceptual adaptation to inverted, reversed, and displaced vision. Psychol. Rev. 72: 419-444.

Harris, C. S. (1980) Insight or out of sight? Two examples of perceptual plasticity in the human adult. In Visual Coding and Adaptability, C. S. Harris, ed., pp. 95-149, Erlbaum, Hillsdale, NJ.

Held, R. (1955) Shifts in binaural localization after prolonged exposures to atypical combinations of stimuli. Am. J. Psychol. 68: 526548.

Jackson, C. V. (1953) Visual factors in auditory localization. Q. J. Exp. Psychol. 5: 52-65. 
Kalil, R., and S. J. Freedman (1967) Compensation for auditory rearrangement in the absence of observer movement. Percept. Mot. Skills 24: 475-478.

Knudsen, E. I. (1982) Auditory and visual maps of space in the optic tectum of the owl. J. Neurosci. 2: 1177-1194.

Knudsen, E. I. (1989) Fused binocular vision is required for development of proper eye alignment in barn owls. Vis. Neurosci. 2: 3540.

Knudsen, E. I., and P. F. Knudsen (1985) Vision guides the adjustment of auditory localization in young barn owls. Science 230: 545-548.

Knudsen, E. I., and P. F. Knudsen (1989a) Visuomotor adaptation to displacing prisms by adult and baby barn owls. J. Neurosci. 9: 32973305.

Knudsen, E. I., and P. F. Knudsen (1989b) Sensitive and critical periods for visually induced changes in sound localization by barn owls. J. Neurosci. (in press).

Knudsen, E. I., G. G. Blasdel, and M. Konishi (1979) Sound localization by the barn owl measured with the search coil technique. J. Comp. Physiol. 133: 1-11.

Knudsen, E. I., S. D. Esterly, and P. F. Knudsen (1984a) Monaural occlusion alters sound localization during a sensitive period in the barn owl. J. Neurosci. 4: 1001-1011.
Knudsen, E. I., P. F. Knudsen, and S. D. Esterly (1984b) A critical period for the recovery of sound localization accuracy following monaural occlusion in the barn owl. J. Neurosci. 4: 1012-1020.

Lackner, J. R. (1973) The role of posture in adaptation to visual rearrangement. Neuropsychologia $11: 33-44$.

Lackner, J. R. (1981) Some aspects of sensory-motor control and adaptation in man. In Intersensory Perception and Sensory Integration, R. D. Walk and H. L. Pisk, eds., Plenum, New York.

Moiseff, A., and M. Konishi (1981) Neuronal and behavioral sensitivity to binaural time differences in the owl. J. Neurosci. 1: 40-48.

Shelton, B. R., and C. L. Searle (1980) The influence of vision on the absolute identification of sound-source position. Perception Psychophysiol. 28: 589-596.

Thurlow, W. R., and T. M. Rosenthal (1976) Further study of existence regions for the "ventriloquism effect." J. Am. Audil. Soc. 1: 280-286.

Warren, D. H., R. B. Welch, and T. J. McCarthy (1982) The role of visual-auditory "compellingness" in the ventriloquism effect: Implications for transitivity among the spatial senses. Perception Psychophysiol. 30: 557-564. 